JOURNAL OF COMPUTATIONAL BIOLOGY

Volume 14, Number 10, 2007

(C) Mary Ann Liebert, Inc.

Pp. 1311-1326

DOI: $10.1089 / \mathrm{cmb} .2006 .0151$

\title{
Bayesian Hierarchical Model for Large-Scale Covariance Matrix Estimation
}

\author{
DONGXIAO ZHU ${ }^{1,3}$ and ALFRED O. HERO III ${ }^{2}$
}

\begin{abstract}
Many bioinformatics problems implicitly depend on estimating large-scale covariance matrix. The traditional approaches tend to give rise to high variance and low accuracy due to "overfitting." We cast the large-scale covariance matrix estimation problem into the Bayesian hierarchical model framework, and introduce dependency between covariance parameters. We demonstrate the advantages of our approaches over the traditional approaches using simulations and OMICS data analysis.
\end{abstract}

Key words: algorithms, computational molecular biology, statistics.

\section{INTRODUCTION}

$\mathbf{E}$ STIMATING A COVARIANCE MATRIX from high-throughput OMICS data is indispensable for many tasks, notably for finding clusters in the data. The problem remains challenging due to the large number of variables $p$ (such as genes or proteins) and the comparatively small number of samples $n$ (such as conditions under which gene expression is measured). The existing approaches that rely on the maximum likelihood estimation or the related unbiased empirical covariance matrix suffer from low accuracy and high variance inherent in any "large $p$, small $n$ " type of data. A regularized and conditioned covariance matrix would be a great improvement over the unconstrained simple estimation of the covariance matrix in the high-throughput OMICS data setting. Estimation of such a matrix is a difficult problem because of inadequate degree of freedom to draw reliable statistical inference on tens of thousands of correlation parameters. Proper constraints need to be imposed on these parameters to overcome this difficulty.

There are two existing approaches. One is based on pairwise correlation estimation followed by variance reduction techniques such as bagging (Hastie et al., 2001) and bootstrap aggregation (Breiman, 1996). Representative work includes the full order-also called Gaussian graphical modeling (GGM)-partial correlation estimation approach (Schafer and Strimmer, 2005a), which introduced a Bayes model from which all correlations are estimated using an empirical Bayes method. Another approach is to obtain improved estimates of the covariance matrix via shrinkage combined with analytic determination of the shrinkage intensity according to the Ledoit-Wolf theorem (Ledoit and Wolf, 2003). The authors showed that the new regularized estimator greatly enhances inferences of gene association networks for synthetic data (Schafer and Strimmer, 2005b). Their approach is based on the assumption that the OMICS data is

\footnotetext{
${ }^{1}$ Stowers Institute for Medical Research, Kansas City, Missouri.

${ }^{2}$ Departments of EECS, Statistics and Biomedical Engineering, University of Michigan, Ann Arbor, Michigan.

${ }^{3}$ Department of Biostatistics, University of Kansas Medical Center, Kansas City, KS.
} 
independently and identically distributed (i.i.d) $p$-variate observations sampled from a $p$-variate Gaussian distribution with the $(p \times p)$ covariance matrix of interest. The assumption is plausible only for small sized homogenous data because the underlying statistical distribution of larger sized heterogenous data is often mixed (Yeung et al., 2001). In both approaches, dependency was introduced among the correlation parameters in different ways.

We advocate the framework of the first set of approaches since it relies on less stringent assumptions, and its usage has been demonstrated by numerous biological examples. We improve over the existing Empirical Bayes method by providing a full Bayesian treatment of the problem. In the Bayesian framework, we derive the posterior distribution for each correlation parameter based on the observed $n \times p$ data matrix. The posterior distributions allow statistical inference of the correlation parameters to be easily drawn.

In our previous work (Zhu et al., 2005a), we described an error control procedure based on a correlation statistic that simultaneously controls statistical and biological significance of the estimated covariance matrix. The correlation statistic works reasonably well for data with relatively large sample size. However, it has poor accuracy for data with small sample size due to overfitting (Ledoit and Wolf, 2004; Schafer and Strimmer, 2005b). Introducing some form of strong dependency among correlation parameters can lead to improved accuracy in this small sample situation. Many approaches to introducing dependency can be adopted. Bayesian hierarchical models accomplish this in a simple but effective manner.

The remainder of the paper is organized into four parts: Bayesian hierarchical model for large-scale covariance matrix estimation (Section 2); simulation studies comparing the Bayesian estimator versus simple estimator (Section 3); analyzing the galactose metabolism data using the proposed Bayesian approach and compared with the traditional approach (Section 4); and conclusion (Section 5).

\section{BAYESIAN HIERARCHICAL MODEL OF COVARIANCE MATRIX}

The framework of Bayesian hierarchical models allows for a high complexity of modelling structure without a large number of parameters (pairwise correlation parameters in this context) (Gelman et al., 2004). We assume the correlation parameters are exchangeable meaning that their joint distribution is invariant to permutations of their indices. This assumption represents a kind of topological invariance that imposes prior assumptions on the location of high correlations in the network. We then regularize variances of the marginal correlation densities by specifying a parent Gaussian distribution from which marginal correlation parameters are sampled. Using a prior population distribution, we are able to introduce dependency into the parameters that tends to avoid problems of overfitting. Using quantiles of posterior distributions of the correlation parameters provides a seamless combination of correlation estimation and strength thresholding that can be used as an alternative to False Discovery Rate-Confidence Interval (FDR-CI) methods (Benjamini and Yekutieli, 2005; Zhu et al., 2005a) for small samples.

Without loss of generality, we employ the marginal correlation coefficient to demonstrate the Bayesian hierarchical model for large-scale (marginal) correlation matrix estimation. The model can be easily extended for large-scale partial correlation matrix estimation, and we will discuss this issue in Section 5. We use $\rho$ to denote the true correlation coefficient between a pair of gene expression profiles (Bickel and Doksum, 2000). Specifically, let $X_{g_{j}(n)}$ be the $n$-th condition index of the $j$-th gene profile and let $S_{X_{g_{i}}, X_{g_{i}}}$, $S_{X_{g_{j}}, X_{g_{j}}}$, and $S_{X_{g_{i}}, X_{g_{j}}}$ be sample variances and covariance defined as:

$$
\begin{aligned}
& S_{X_{g_{i}}, X_{g_{i}}}=(N-1)^{-1} \sum_{n=1}^{N}\left(X_{g_{i}(n)}-\overline{X_{g_{i}}}\right)^{2}, \\
& S_{X_{g_{j}}, X_{g_{j}}}=(N-1)^{-1} \sum_{n=1}^{N}\left(X_{g_{j}(n)}-\overline{X_{g_{j}}}\right)^{2}, \\
& S_{X_{g_{i}}, X_{g_{j}}}=(N-1)^{-1} \sum_{n=1}^{N}\left(X_{g_{i}(n)}-\overline{X_{g_{i}}}\right)\left(X_{g_{j}(n)}-\overline{X_{g_{j}}}\right) .
\end{aligned}
$$




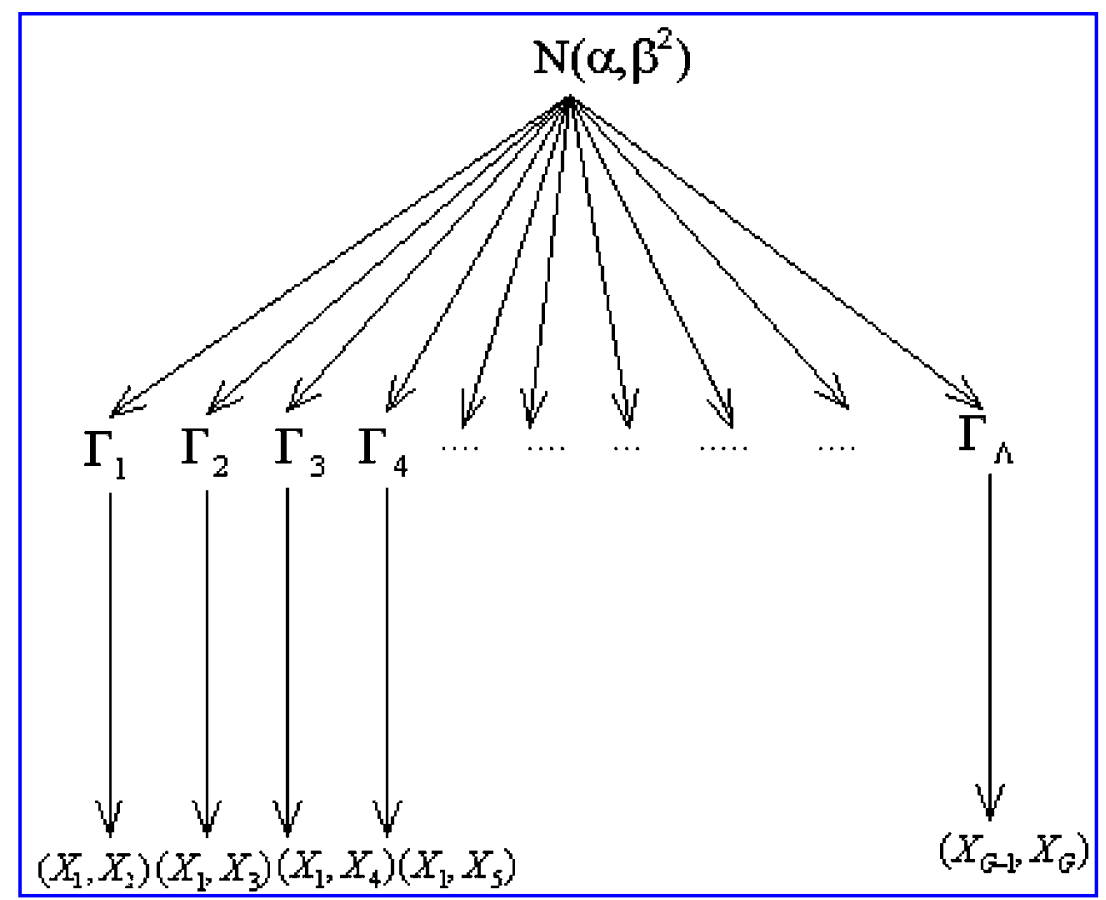

FIG. 1. Bayesian hierarchical model structure (Gelman et al., 2004).

The true correlation coefficient is defined as

$$
\rho=\frac{\mathrm{E}\left[S_{X_{g_{i}}, X_{j}}\right]}{\sqrt{\mathrm{E}\left[S_{X_{g_{i}}, X_{g_{i}}}\right] \mathrm{E}\left[S_{X_{g_{j}}, X_{g_{j}}}\right]}},
$$

where E[.] is statistical expectation. For $G$ gene expression profiles in a gene microarray sequence, there are $\Lambda=\left(\begin{array}{c}G \\ 2\end{array}\right)$ of these correlation parameters $\rho$ that need to be estimated, denoted as $\rho_{\lambda}, \lambda=1, \ldots, \Lambda$. We define $\hat{\rho}_{\lambda}$ as the $\lambda$ th sample correlation coefficient, and $\hat{\Gamma}_{\lambda}$ as the hyperbolic arc-tangent transformation of $\hat{\rho}_{\lambda}$. Then the transformed sample correlation coefficient $\hat{\Gamma}_{\lambda}=\operatorname{atanh}\left(\hat{\rho}_{\lambda}\right)$ is asymptotically Gaussian distributed with means of $\rho_{\lambda}$ and stabilized variance approximations of $\sigma_{\lambda}^{2}=1 /(N-3)$ (Fisher, 1923). Here $N$ is the sample size. We define $\Gamma_{\lambda}=\operatorname{atanh}\left(\rho_{\lambda}\right)$ as the corresponding transformed true correlation coefficient.

Our previous simulation studies showed that this variance approximation works reasonably well even at a relatively small sample size (e.g., $N<10$ ) (Zhu et al., 2005a). In this sequel we assume known variance of the transformed correlation matrix to reduce the computational complexity of the full Bayesian correlation matrix estimation. In cases of unknown variances, the conditional posterior distribution can not generally be written in closed form. For this reason, Markov Chain Monte Carlo (MCMC) techniques might be applied but at high computational cost.

From our assumption that the $\left\{\rho_{\lambda}\right\}_{\lambda=1}^{\Lambda}$ are exchangeable we model $\left\{\rho_{\lambda}\right\}_{\lambda=1}^{\Lambda}$ as random variables drawn from a Gaussian distribution with unknown hyperparameters $\left(\alpha, \beta^{2}\right.$; Fig. 1).

$$
p\left(\Gamma_{1}, \ldots, \Gamma_{\Lambda} \mid \alpha, \beta^{2}\right)=\prod_{\lambda=1}^{\Lambda} P\left(\Gamma_{\lambda} \mid \alpha, \beta^{2}\right),
$$

where $P\left(\Gamma_{\lambda} \mid \alpha, \beta^{2}\right)$ is a Gaussian distribution with mean $\alpha$ and variance $\beta^{2}$.

In order to generate conditional posterior distributions $p\left(\Gamma_{\lambda} \mid \alpha, \beta, y\right)$ for each parameter $\Gamma_{\lambda}, \lambda=$ $1, \ldots, \Lambda$, where $y$ represents a crude estimate of correlation (e.g. using Pearson correlation coefficient) throughout this article, we performed simulation steps as follows (Gelman et al., 2004) (refer to Appendix for details): 
- Assign prior distribution for $\beta$, e.g. uniform prior distribution $p(\beta) \propto 1$. Note, the choice of uniform prior yields a proper posterior density while other noninformative prior distributions such as, $p(\beta) \propto \beta^{-1}$ do not (refer to the Appendix for mathematical proof).

- Draw $\beta$ from posterior distribution $p(\beta \mid y)$.

$$
\begin{gathered}
p(\beta \mid y) \propto \frac{p(\beta) \prod_{\lambda=1}^{\Lambda} N\left(\widehat{\Gamma}_{\lambda} \mid \hat{\alpha}, \sigma_{\lambda}^{2}+\beta^{2}\right)}{N\left(\hat{\alpha} \mid \hat{\alpha}, V_{\alpha}\right)} \\
\propto p(\beta) V_{\alpha}^{1 / 2} \prod_{\lambda=1}^{\Lambda}\left(\sigma_{\lambda}^{2}+\beta^{2}\right)^{-1 / 2} \exp \left(-\frac{\left(\widehat{\Gamma}_{\lambda}-\widehat{\alpha}\right)^{2}}{2\left(\sigma_{\lambda}^{2}+\beta^{2}\right)}\right),
\end{gathered}
$$

where $\hat{\alpha}$ and $V_{\alpha}$ are defined as:

$$
\hat{\alpha}=\frac{\sum_{\lambda=1}^{\Lambda} \frac{1}{\sigma_{\lambda}^{2}+\beta^{2}} \hat{\Gamma}_{\lambda}}{\sum_{\lambda=1}^{\Lambda} \frac{1}{\sigma_{\lambda}^{2}+\beta^{2}}},
$$

and

$$
V_{\alpha}^{-1}=\sum_{\lambda=1}^{\Lambda} \frac{1}{\sigma_{\lambda}^{2}+\beta^{2}} .
$$

See the Appendix for detailed derivation of $p(\beta \mid y)$.

- Draw $\alpha$ from $p(\alpha \mid \beta, y)$. Combining the data with the uniform prior density $p(\alpha \mid \beta)$ yields,

$$
p(\alpha \mid \beta, y) \sim N\left(\hat{\alpha}, V_{\alpha}\right)
$$

where $\hat{\alpha}$ is a precision-weighted average of the $\hat{\Gamma} \mathrm{s}$ and $V_{\alpha}$ is the total precision. Note, we define precision as inverse of variance.

- Draw $\Gamma_{\lambda}$ from $p\left(\Gamma_{\lambda} \mid \alpha, \beta, y\right)$

$$
p\left(\Gamma_{\lambda} \mid \alpha, \beta, y\right) \sim N\left(\hat{\Theta}_{\lambda}, V_{\lambda}\right)
$$

where $\hat{\Theta}_{\lambda}, V_{\lambda}$ are defined as:

$$
\hat{\Theta}_{\lambda}=\frac{\frac{1}{\sigma_{\lambda}^{2}} \hat{\Gamma}_{\lambda}+\frac{1}{\beta^{2}} \alpha}{\frac{1}{\sigma_{\lambda}^{2}}+\frac{1}{\beta^{2}}}
$$

and

$$
V_{\lambda}=\frac{1}{\frac{1}{\sigma_{\lambda}^{2}}+\frac{1}{\beta^{2}}}
$$

The atanh-transformed posterior mean correlation coefficient $\hat{\Theta}_{\lambda}$ is a precision-weighted average of the prior population mean $\alpha$ and the $\lambda$ th sample mean $\hat{\Gamma}_{\lambda}$. 
The posterior distribution (Eq. (8)) contains all the current information about the atanh-transformed parameter $\rho_{\lambda}$. In particular, the posterior mean and posterior confidence interval can be derived. The posterior mean is

$$
\begin{aligned}
\mathrm{E}\left[\Gamma_{\lambda} \mid y\right] & =\mathrm{E}\left[\operatorname{atanh}\left(\rho_{\lambda}\right) \mid y\right] \\
& =\operatorname{avg}\left(\hat{\Gamma}_{\lambda}\right) .
\end{aligned}
$$

For deriving the posterior interval of the $\rho_{\lambda}$, we used the fact that the cumulative density function (CDF) of $\Gamma_{\lambda}^{\prime}=\frac{\Gamma_{\lambda}-\hat{\Theta}_{\lambda}}{\sqrt{V_{\lambda}}}$ is $\Phi$, the cdf of standard Gaussian random variable. Hence, we define its quantile function as $\Phi^{-1}$, and write the $(1-q) \times 100 \%$ posterior interval of the parameter $\Gamma_{\lambda}{ }^{\prime}$ :

$$
I^{\Gamma_{\lambda}{ }^{\prime}}(q)=\left[\Phi^{-1}(q / 2), \Phi^{-1}(1-q / 2)\right] .
$$

After some algebra derivation and based on the fact that tanh is a monotonically increasing function, we have a $(1-q) \times 100 \%$ posterior confidence interval for the parameter $\rho_{\lambda}$ :

$$
I^{\rho_{\lambda}}(q)=\left[\tanh \left(\sqrt{V_{\lambda}}\left(\Phi^{-1}(q / 2)\right)+\hat{\Theta}_{\lambda}\right), \tanh \left(\sqrt{V_{\lambda}}\left(\Phi^{-1}(1-q / 2)\right)+\hat{\Theta}_{\lambda}\right)\right] .
$$

\section{SIMULATION STUDIES}

\subsection{Comparisons in terms of confidence interval, mean squared error, and variance}

We evaluated the performance of full Bayesian hierarchical model estimation of correlations and compared with the frequentist method in Zhu et al. (2005a). We define the frequentist confidence interval (CI) as follows: If $\mathrm{L}$ and $\mathrm{U}$ are statistics (i.e., observable random variables) whose probability distribution depends on some unobservable parameter $\theta$, and

$$
\operatorname{Pr}(L \leq \theta \leq U)=q, q \in(0,1)
$$

then the random interval $[\mathrm{L}, \mathrm{U}]$ is a $(1-q) \times 100 \%$ confidence interval for $\theta$. A frequentist confidence interval may strictly be interpreted only in relation to a sequence of similar inferences that might be made in repeated trials, while a Bayesian (confidence) interval for an unknown quantity of interest can be directly regarded as having a high probability of containing the unknown quantity. Therefore, the Bayesian approach, where a reliable prior is available, facilitates a common-sense interpretation of statistical conclusions (Gelman et al., 2004).

We first compared two point estimators of correlations in terms of the average width of the individual frequentist (Pearson) CIs for the correlation parameters versus that of the posterior CIs for the same set of correlation parameters at the corresponding significance levels. Obviously, more concentrated (narrower) CIs, at the given significance level, are superior to less concentrated CIs. It is clear from Figures 2 and 3 that the average Bayesian posterior CIs are uniformly narrower than the average freqentist CIs in both small $(N=4)$ and larger sample data $(N=20)$. This dramatic contrast indicates the advantages of the Bayesian approach for small sample size problems (Fig. 3). From Eqs. (21) and (3), the posterior distributions of the mean $p(\alpha \mid \beta, y)$ and of the variance $p(\beta \mid y)$ are decreasing functions of $\Lambda$, i.e., the number of correlation parameter $\Gamma$ s. Therefore, narrower posterior CIs are expected for larger $\Lambda$. On the other hand, wider CIs are expected when transforming individual frequentist CIs into simultaneous FDR-CIs.

We also compared these two correlation estimators in terms of mean squared error (MSE) and variance criteria. Similar to the definition in Zhu et al. (2005a), the MSE is defined as:

$$
\left.\operatorname{MSE}\left(\hat{\rho}_{\lambda}\right)=\mathrm{E}(\hat{\rho}-\rho)^{2}\right)=\frac{1}{\Lambda} \sum_{\lambda=1}^{\Lambda}\left(\hat{\rho}_{\lambda}-\rho_{\lambda}\right)^{2},
$$

where $\rho_{\lambda}$ is the true population correlation, and $\hat{\rho}_{\lambda}$ is the sample correlation estimator, $\lambda$ is the parameter index, and $\Lambda$ is the total number of parameters. 


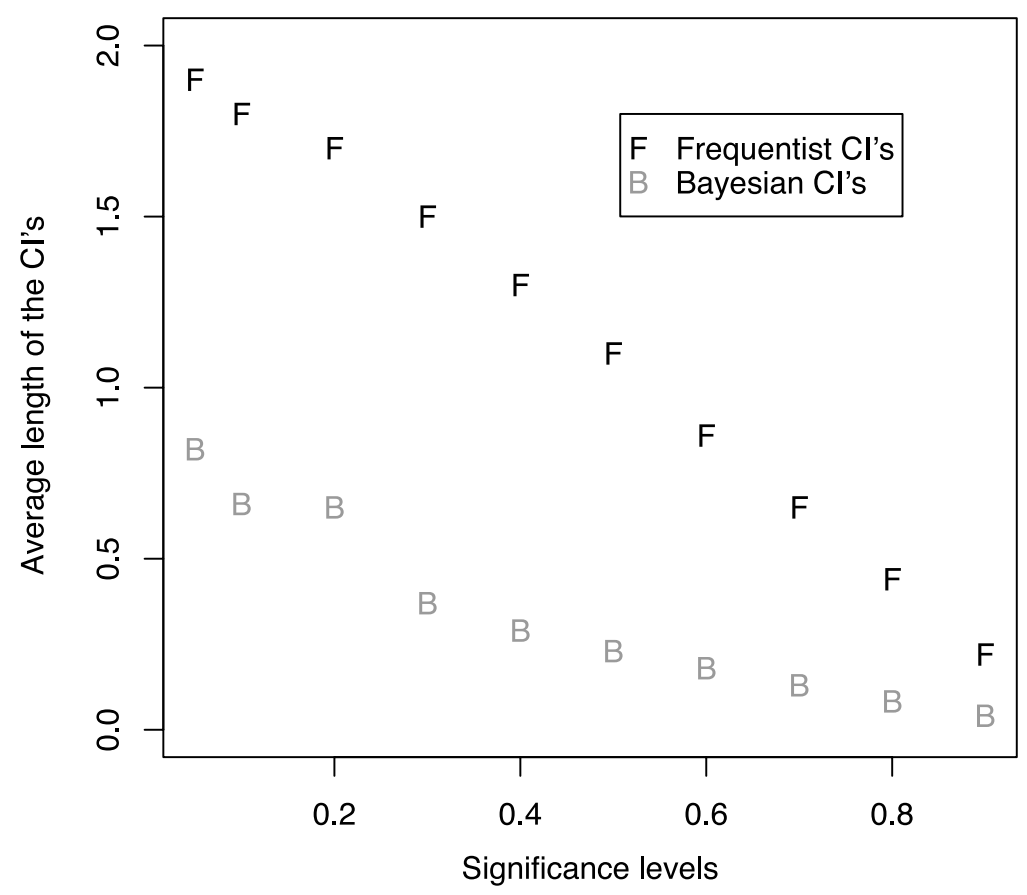

FIG. 2. Comparison of average posterior confidence intervals (CIs) versus average individual frequentist CIs over a wide range of significance levels at a small sample size $(n=4)$.

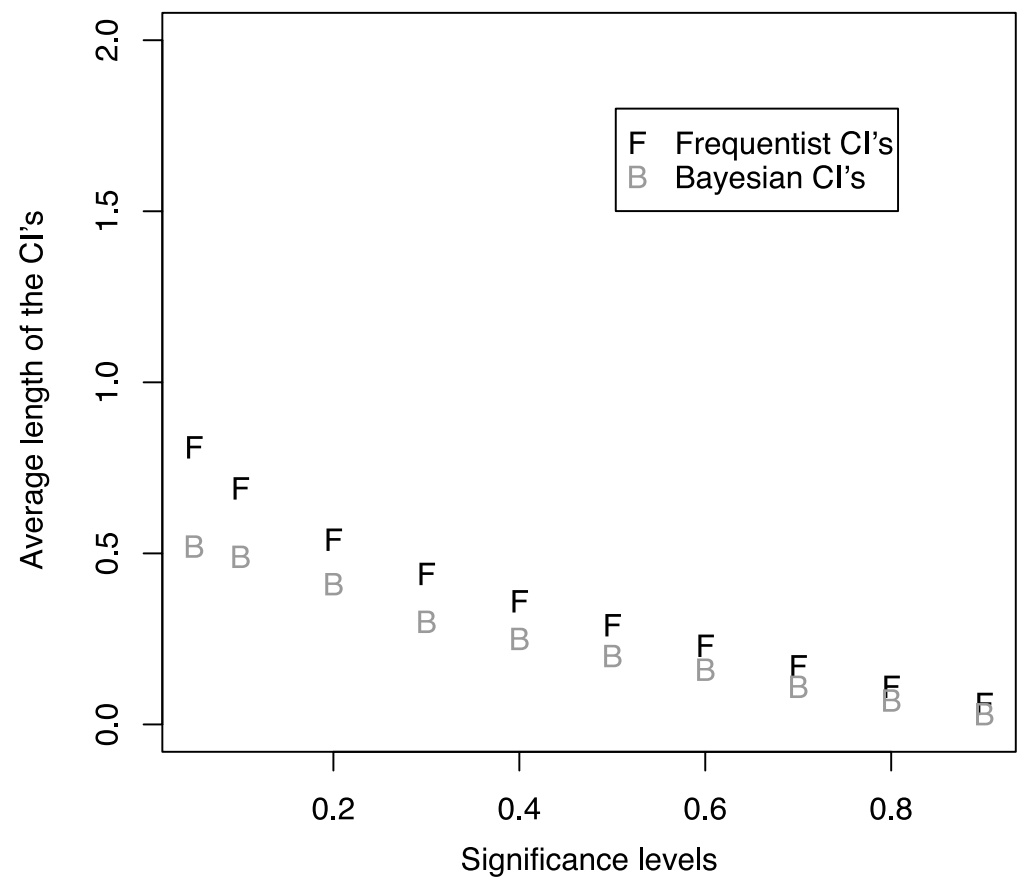

FIG. 3. Comparison of average posterior confidence intervals (CIs) versus average individual frequentist CIs over a wide range of significance levels at a larger sample size $(n=20)$. 

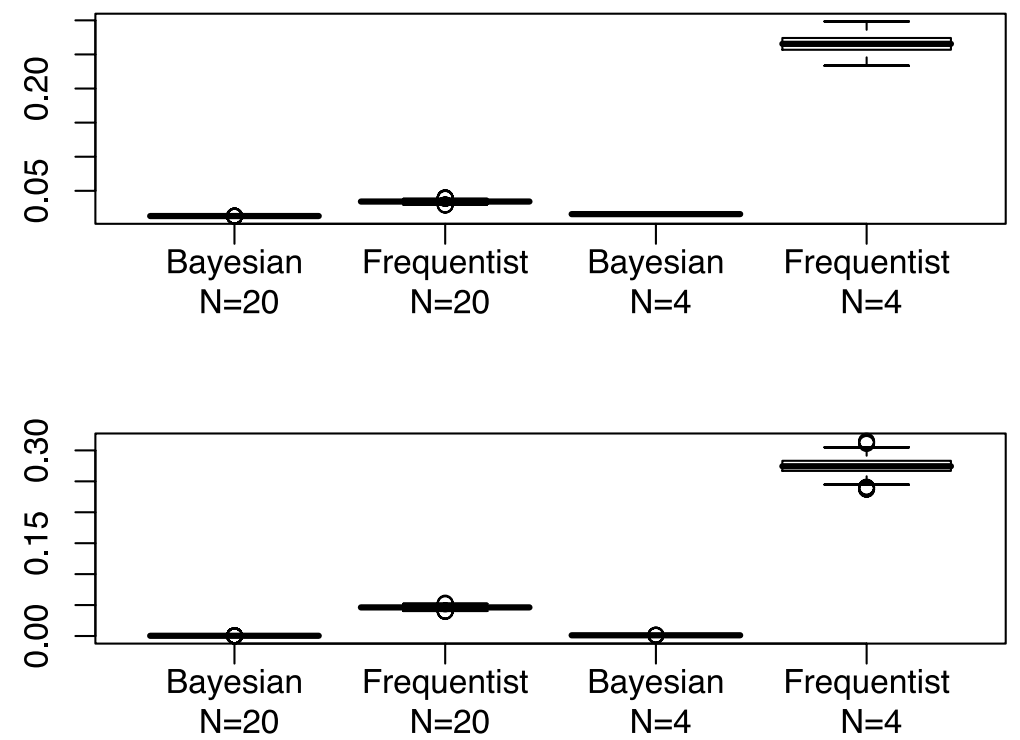

FIG. 4. Mean squared errors (MSEs) (upper) and variances (lower) of the Bayesian estimations versus the frequentist estimations over 500 runs of simulations.

The simulation steps proceed as follows:

- Draw $\Lambda$ population correlations from a normal distribution with known mean $(\alpha)$ and variance $(\beta)$ (hyperparameters) as defined in Eq. (2).

- Re-estimate the $\Lambda$ parameters either separately using the frequentist (Pearson) correlation estimator or using the Bayesian hierarchical model estimator. For the Bayesian approach, the correlation estimator is the posterior mean (Eq. (11)).

- Compare the two estimators in terms of both MSE and variance. An estimator with low MSE and variance is considered to be superior.

Figure 4 plots MSEs (upper panel) and variances (lower panels) of Bayesian correlation estimators and frequentist (Pearson) correlation estimators at a small sample size (e.g., $N=4$ ) and a larger sample size (e.g., $N=20$ ) over 500 runs of simulations. It is evident in the upper panel of Figure 4 that the MSE of Bayesian estimators is about three-fold smaller than the frequentist estimators for the larger sample size. Similarly to the CIs comparisons, this indicates the advantages of the Bayesian correlation estimator for small sample size problems (Fig. 4). The lower panel of Figure 4 plots variances of the Bayesian correlation estimator and the frequentist correlation estimator. Again, the comparison of variances follow the same trend as that of the MSEs (Fig. 4).

It is worth mentioning that the above simulations assumed a known Bayesian hierarchical model. In order to test the robustness of our algorithm to model mismatch, we also generated data using uniform distribution but implemented with Pearson CIs and Bayesian CIs that assume mismatched Gaussian and hierarchical models, respectively. In Figure 5, we compared the average width of individual Pearson CIs with that of individual Bayesian intervals. The superior performance of the hierarchical Bayesian estimator (Figs. 2 and 3) is clearly offset by the invalid model assumption in that average Bayesian CIs are slightly wider than average frequentist CIs (Fig. 5). This simulation result reflects the importance of the Fisher transformation.

\subsection{Evaluation of the Bayesian hierarchical model}

In order to evaluate our Bayesian approach in terms of error control and compare with the frequentist counterpart, we simulated pairwise gene expression data based on known population covariances, and then 


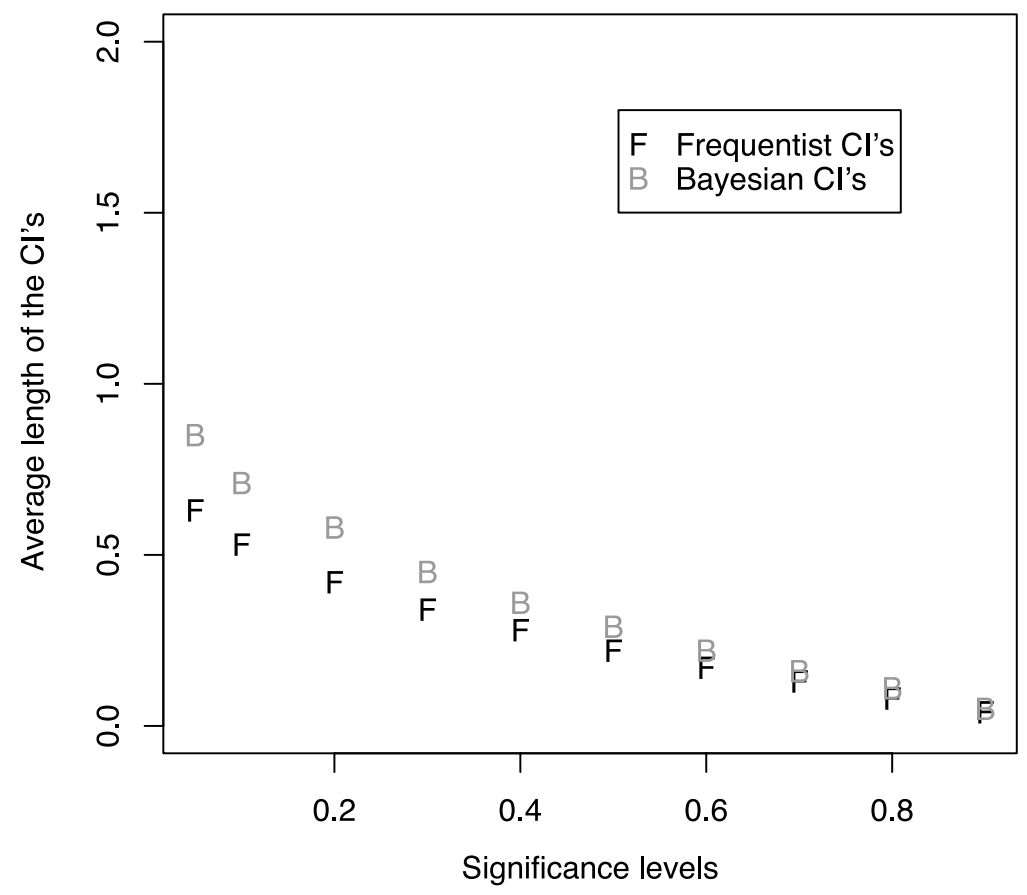

FIG. 5. Comparison of average confidence intervals (CIs) when the Bayesian model is unsustained.

simulated Bayesian confidence intervals for each parameter from the hierarchical model. The actual false positive (FP) at a given minimal acceptable strength (MAS) level is calculated as a ratio of the number of screened gene pairs whose corresponding population correlation parameters $\rho_{i, j}$ are less than the MAS level specified, divided by the total number of gene pairs. The actual MAS is the minimum true discovery of population correlation $\rho_{i, j}$ among the screened pairs. We specified 16 pairs of (FP,MAS) criteria (four FP levels: $0.2,0.4,0.6,0.8$; four MAS levels: $0.2,0.4,0.6,0.8$ ), and each is plotted as a different upper case Roman alphabet (red) in Figure 6. The 16 corresponding pairs of actual (FP,MAS) criteria are also shown in Figure 6 using the same set of lower case Roman alphabets (Blue). It can be observed that generally the actual FPs (lower case) fall further below the specified constraint (upper case) than those in Figure 4 of Zhu et al. (2005a) (Fig. 6) did, and the actual MASs (lower case) fall above the specified constraints (upper case). The more dramatic deviations of actual FPs from their specified levels are due to multiple factors, such as, lack of multiplicity adjustment and the conservative asymptotic approximation made in Zhu et al. (2005a). Simulations using some other combinations of $N$ and $\Lambda$, as compared with the FDR-CI approach, give rise to similar results. We conclude that the Bayesian hierarchical model yields better correlation estimates. However, the false positive rate is overestimated by the Bayesian procedure and this leads to overly stringent error control.

\section{APPLICATIONS TO NETWORK CONSTRUCTION AND SEEDED CLUSTERING}

\subsection{Constructing relevance networks}

We applied the Bayesian hierarchical model to high-throughput data and compared it with the frequentist approach using the same subset of yeast galactose catabolism two-color microarray data that was described in Zhu et al. (2005a). The data contains 997 gene expression profiles across 20 genetic/physiological conditions that was identified by Ideker et al using the generalized likelihood ratio test (Ideker et al., 2000). 


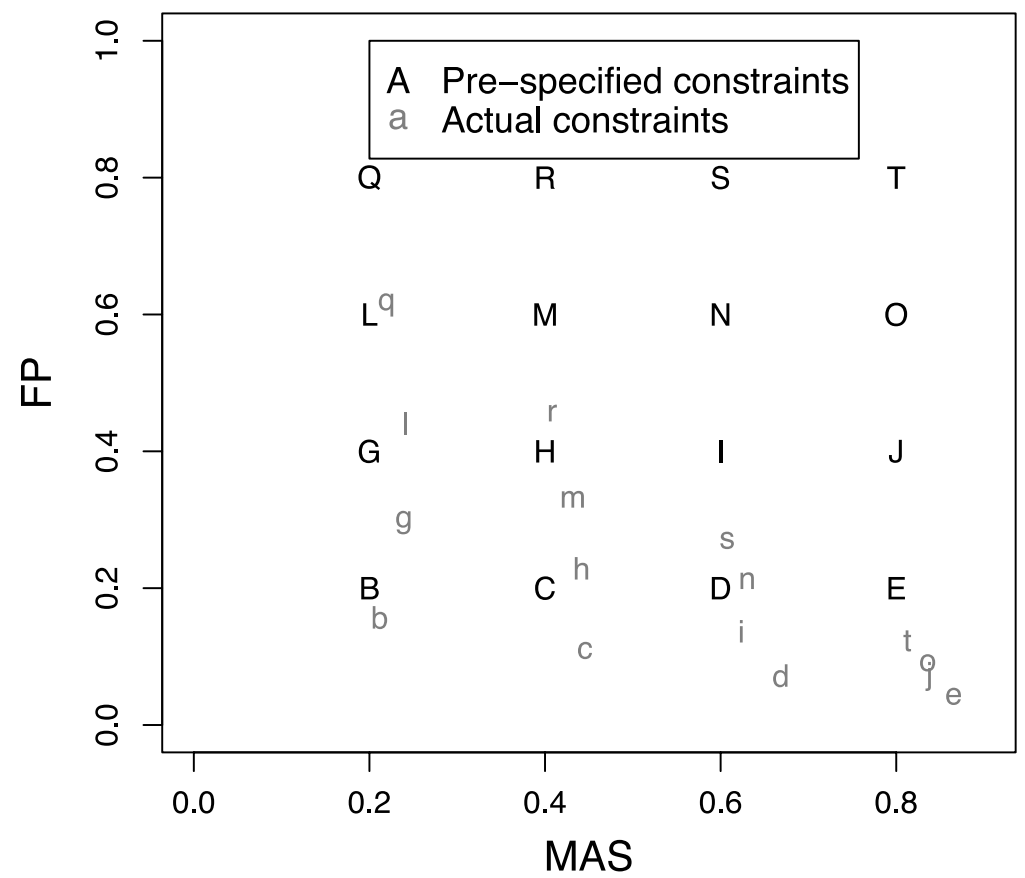

FIG. 6. Evaluation of error control of the Bayesian hierarchical model. Sample size $n=20$, and $\Lambda=1000$ correlation coefficients were simulated. Simulations using smaller sample size data yield more stringent error control.

Following the procedure described in Section 2, we simulated the empirical posterior distribution for each of the $\left(\begin{array}{c}997 \\ 2\end{array}\right)=496,506$ correlation parameters $\rho_{\lambda}$. The $(1-q) \times 100 \%$ posterior interval for each "parameter" was obtained by thresholding $q / 2 \times 100 \%$ and $(1-q / 2) \times 100 \%$ of its quantile function (Eq. (13)). Analogous to the FDR-CI screening procedure described in Zhu et al. (2005a), a network edge is declared to be present at the significance level $q$ and the MAS level cormin if its posterior CI does not intersect with [-cormin, cormin]. We sought to compare the two approaches in terms of network topological properties that are interesting to biologists. In particular, we compared the biological functional annotations of the top hub genes of the two networks. In Zhu et al. (2005a), we controlled FDR at 5\%, and constructed networks at five MAS levels, i.e., 0.5, 0.6, 0.7, 0.8, 0.9. Correspondingly, 18,135, 9337, $4151,1346,133$ edges were declared to be present using Pearson correlation statistic alone. Controlling the significance level at 5\%, we screened the same set of numbers of edges using the Bayesian hierarchal model to construct the five networks that are more comparable to those in Zhu et al. (2005a). A list of stable hub genes was obtained by calculating and sorting the average rank of each vertex (gene) degree over five networks (Table 1).

Comparing Table 1 with that reported in Zhu et al. (2005a), note that the GO biological process annotation "protein biosynthesis [GO:0006412]" and/or its children annotations "hypusine biosynthesis [GO:0046515]," "branched chain family amino acid biosynthesis [GO:0009082]," and "tryptophan biosynthesis [GO:0000162]" are significantly enriched in both tables. This is consistent with the established fact that protein biosynthesis plays a key role in galactose metabolism (Berg et al., 2006). The underlying biological mechanism is that many types of proteins need to be synthesized upon switching from primary carbon source (glucose) to secondary carbon source (galactose) or the other way around (Wieczorke et al., 1999).

A salient feature in Table 1 that differs from that of Zhu et al. (2005a) is that it includes several transporters and regulators such as GAP1 [GO:0006865], YBR043C [GO:0006855], and ASC1 [GO:0006417]. These proteins are essential for a smooth transition from glucose to galactose (Berg et al., 2006; Wieczorke et al., 1999). In addition, Table 1 also includes several uncharacterized genes that are hypothesized to be important for galactose metabolism. In general, the Bayesian data analysis results not only conform to the previous frequentist data analysis results, but also provide additional justification for the biological mechanism and motivation for illustrating new gene functions. 
Table 1. Top Twenty “Hub Genes” from Bayesian Hierarchical Model Applied to the Galactose Metabolism Data (IDEker Et Al., 2000)

\begin{tabular}{lcl}
\hline Gene name & Average rank & \multicolumn{1}{c}{ GO annotation } \\
\hline YJR070C & 4 & Hypusine biosynthesis [GO:0046515] \\
YBR043C & 4.4 & Multidrug transport [GO:0006855] \\
AGA2 & 4.4 & Agglutination [GO:0000771] \\
RPP0 & 4.6 & Protein biosynthesis [GO:0006412] \\
RPL26A & 4.6 & Protein biosynthesis [GO:0006412] \\
YOR263C & 5 & Biological process unknown \\
TRP2 & 5.4 & Tryptophan biosynthesis [GO:0000162] \\
ASC1 & 5.6 & Regulation of protein biosynthesis [GO:0006417] \\
YIL064W & 5.6 & Biological process unknown \\
BOP2 & 5.6 & Biological process unknown \\
GAP1 & 5.8 & Amino acid transport [GO:0006865] \\
RPS2 & 6 & Protein biosynthesis [GO:0006412] \\
RPL11A & 6.2 & Protein biosynthesis [GO:0006412] \\
SSF2 & 6.2 & Ribosomal subunit assembly [GO:0042257] \\
ILV5 & 6.2 & Branched chain family amino acid biosynthesis [GO:0009082] \\
YPL185W & 6.2 & Biological process unknown \\
PCK1 & 6.4 & Hexose biosynthesis [GO:0019319] \\
YDR100W & 6.4 & Biological process unknown \\
YMR291W & 6.6 & Biological process unknown \\
ATC1 & 6.6 & Bipolar bud site selection [GO:0007121] \\
\hline
\end{tabular}

The rank of each gene is the average rank over five different networks with the same set of edge numbers as in Table 1 of Zhu et al. (2005a). The highest ranked gene is the most connected and stable gene under varying constraints of (FP,MAS).

FP, false positive; MAS, minimal acceptable strength.

\subsection{Seeded clustering}

In parallel with the application of the two-stage algorithm to rediscover the galactose metabolic pathway reported in Zhu et al. (2005a), we also applied the Bayesian hierarchical model to perform the seeded (one-to-all) clustering. Performance was evaluated according to the relative ranks of a handful of known members of the galactose metabolic pathway. The gene ranks were reported instead of $p$-values due to substantial differences of the two statistical frameworks.

We selected gene "GAL10" as the "seed gene" in order to compare the results with those reported in Zhu et al. (2005a). The comparison was made at a large sample size $N=20$ and a smaller sample size $N=4$, respectively, aiming to examine the performance of the two methods as a function of the sample size. In the former, we used all the 20 genetic/physiological conditions under which gene expression levels were measured (Table 2). In the latter, we sampled a small subset (e.g., $N=4$ ) of these 20 conditions each time without replication and repeated a number of times to obtain a "bagged" (stable) estimation of gene ranks in the seeded clusters (Table 2).

When all the 20 observations were used, the two approaches gave rise to very similar seeded clusters indicating that the Bayesian hierarchial model approach is as powerful as the frequentist approach for relatively large sample size problems. As shown in Table 2, all of the top 20 seeded gene pairs have identical ranks across two methods. When multiple random subsets of the data were used, many genes had dissimilar average ranks across the two approaches. Among the top five genes (GAL10, GAL7, GCY1 GAL1, GAL2) screened by the seeded clustering using "GAL10" as the seed gene (Zhu et al., 2005a) (Table 3), four out of five (GAL10, GAL7, GAL1, GAL2) genes ranked higher in Bayesian estimation than those in frequentist estimation, and the remaining "GCY1" gene received tie ranks. See supplemental figures (Figures S-1 and S-2) for comparing untransformed and transformed example posterior distributions. In addition, our results provide strong experimental motivation for examining the genes that received higher 
TABLE 2. COMPARISON OF BAYESIAN ESTIMATIONS VERSUS FREQUENTIST ESTIMATIONS Using "Seeded” Clustering at SMall and Larger Sample Sizes

\begin{tabular}{|c|c|c|c|c|c|c|c|}
\hline \multicolumn{4}{|c|}{$n=4$} & \multicolumn{4}{|c|}{$n=20$} \\
\hline Gene 1 & Gene 2 & Bayesian & Frequentist & Gene 1 & Gene 2 & Bayesian & Frequentist \\
\hline GAL10 & GAL1 & 5.25 & 5.35 & GAL10 & GAL7 & 1 & 1 \\
\hline GAL10 & GAL2 & 6.65 & 7.4 & GAL10 & GCY1 & 2 & 2 \\
\hline GAL10 & GAL7 & 6.7 & 6.85 & GAL10 & GAL1 & 3 & 3 \\
\hline GAL10 & GCY1 & 7.7 & 7.7 & GAL10 & GAL2 & 4 & 4 \\
\hline GAL10 & YOR121C & 8.05 & 7.8 & GAL10 & YOR121C & 5 & 5 \\
\hline GAL10 & YEL057C & 8.55 & 10.6 & GAL10 & YEL057C & 6 & 6 \\
\hline GAL10 & SSU1 & 8.6 & 7.65 & GAL10 & YDR010C & 7 & 7 \\
\hline GAL10 & FKS1 & 8.75 & 8.25 & GAL10 & SSU1 & 8 & 8 \\
\hline GAL10 & PCL10 & 9.95 & 7.85 & GAL10 & PCL10 & 9 & 9 \\
\hline GAL10 & YJL212C & 11 & 8.85 & GAL10 & YJL212C & 10 & 10 \\
\hline GAL10 & MET14 & 11.1 & 10.4 & GAL10 & FKS1 & 11 & 11 \\
\hline GAL10 & YDR010C & 11.3 & 10.9 & GAL10 & MET14 & 12 & 12 \\
\hline GAL10 & MCM1 & 11.35 & 12.3 & GAL10 & MCM1 & 13 & 13 \\
\hline GAL10 & EXG1 & 11.85 & 13.1 & GAL10 & EXG1 & 14 & 14 \\
\hline GAL10 & CRH1 & 12.05 & 12.95 & GAL10 & ARG1 & 15 & 15 \\
\hline GAL10 & ARG7 & 12.8 & 12.3 & GAL10 & CRH1 & 16 & 16 \\
\hline GAL10 & YPR157W & 13.2 & 15.35 & GAL10 & PRY2 & 17 & 17 \\
\hline GAL10 & PRY2 & 14.4 & 13.3 & GAL10 & YPR157W & 18 & 18 \\
\hline GAL10 & YKR012C & 14.6 & 16.25 & GAL10 & YKR012C & 19 & 19 \\
\hline GAL10 & CPA2 & 16.15 & 14.85 & GAL10 & CPA2 & 20 & 20 \\
\hline
\end{tabular}

In the former, the ranks were averaged over 100 estimations, in each of which a subset data of sample size $n=4$ was randomly sampled from the whole data of sample size $n=20$. In the latter, the ranks were obtained using the whole data of sample size $n=20$. Known genes in the pathway are in bold face.

Table 3. Clustering Co-Expressed Genes with Bayesian Hierarchical Model at the SignificAnCE LeVEl 5\% USing "GAL10" AS THE "SEed Gene"

\begin{tabular}{lllll}
\hline Gene 1 & Gene 2 & \multicolumn{1}{c}{$2.5 \%$} & \multicolumn{1}{c}{$50 \%$} & $97.5 \%$ \\
\hline GAL10 & GAL7 & 0.699967273 & 0.843269806 & 0.919377659 \\
GAL10 & GCY1 & 0.695895931 & 0.83904824 & 0.917448689 \\
GAL10 & GAL1 & 0.685628575 & 0.824914454 & 0.906837751 \\
GAL10 & GAL2 & 0.664031223 & 0.817631953 & 0.903466008 \\
GAL10 & YOR121C & 0.652511568 & 0.814118521 & 0.901500909 \\
GAL10 & YDR010C & 0.574348042 & 0.77081336 & 0.875409524 \\
GAL10 & YEL057C & 0.582835775 & 0.769743768 & 0.880618535 \\
GAL10 & SSU1 & 0.584487078 & 0.769335123 & 0.879019784 \\
GAL10 & PCL10 & 0.552529392 & 0.751817344 & 0.871763977 \\
GAL10 & YJL212C & 0.543601479 & 0.747480187 & 0.862433646 \\
GAL10 & MET14 & 0.525320838 & 0.723128249 & 0.852859396 \\
GAL10 & FKS1 & 0.515021843 & 0.719874179 & 0.854759107 \\
GAL10 & MCM1 & 0.474061933 & 0.697313988 & 0.834101087 \\
GAL10 & EXG1 & 0.446476056 & 0.666889754 & 0.818233838 \\
GAL10 & ARG1 & 0.382292245 & 0.63708452 & 0.807736956 \\
GAL10 & CRH1 & 0.344971636 & 0.594425382 & 0.773435199 \\
GAL10 & PRY1 & 0.299057555 & 0.588919717 & 0.774038296 \\
GAL10 & YPR157W & 0.29645952 & 0.576125639 & 0.765975044 \\
GAL10 & CPA2 & 0.303356019 & 0.571475575 & 0.745218878 \\
GAL10 & YKR012C & 0.262900828 & 0.566724743 & 0.748081117 \\
\hline
\end{tabular}

Known genes in the pathway are in bold face $(n=20)$. 
Untransformed Posterior Distribution

$(\mathrm{N}=\mathbf{2 0})$

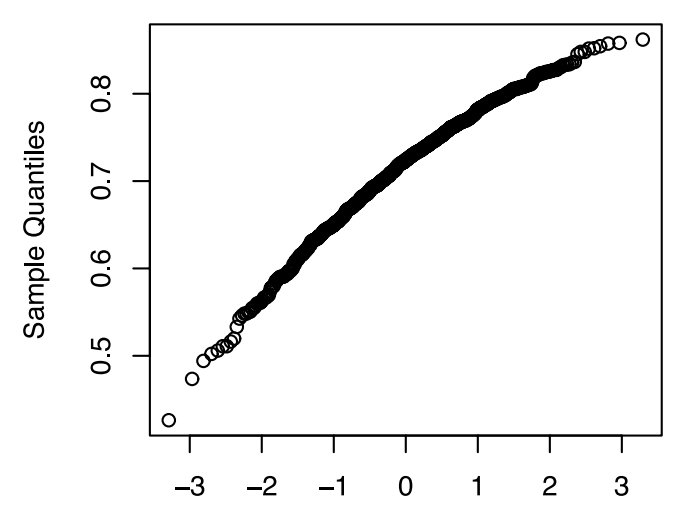

Theoretical Quantiles

\section{Untransformed Posterior Distribution} $(\mathrm{N}=4)$

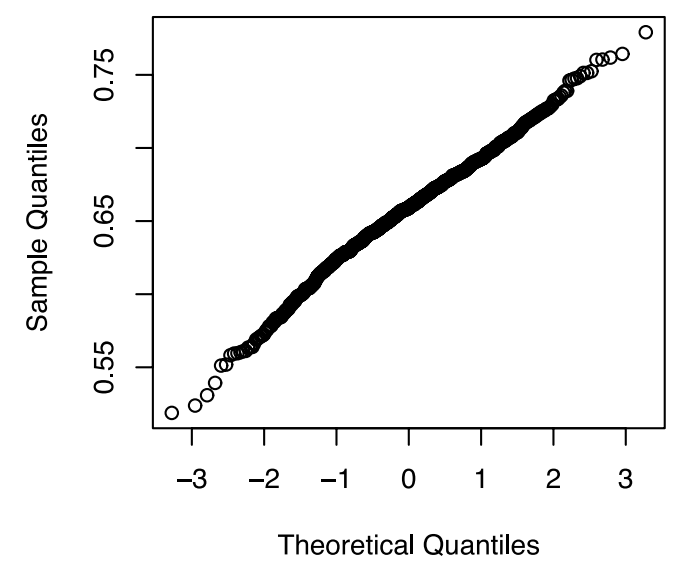

Transformed Posterior Distribution $(\mathrm{N}=\mathbf{2 0})$

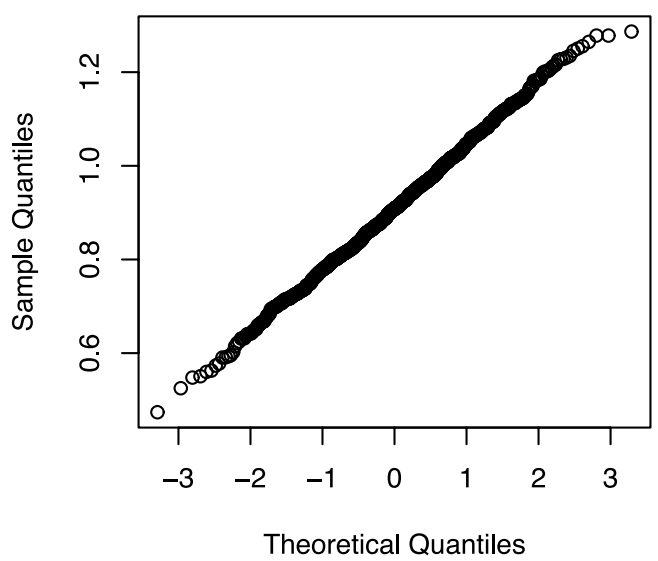

Transformed Posterior Distribution $(\mathrm{N}=4)$

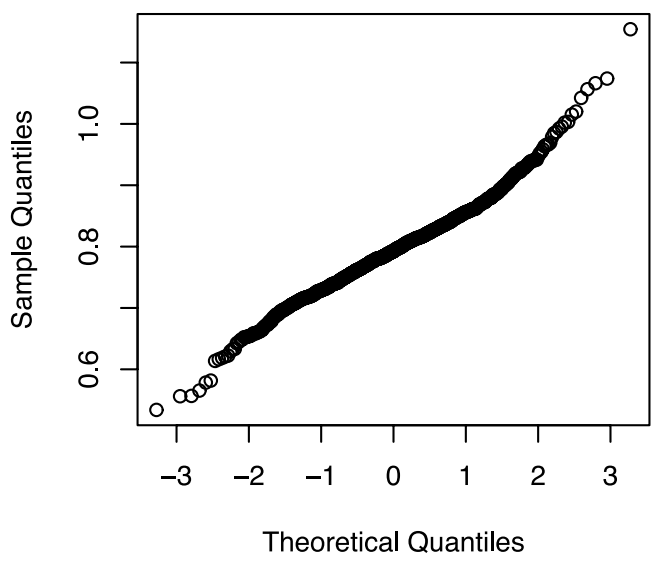

FIG. S-1. Transformed and untransformed posterior distributions at small and large sample size (Example 1).

ranks in the Bayesian analysis, for example, gene YEL057C. The evaluation using "GAL7" as the "seed gene" gave similar results.

\section{CONCLUSION}

Numerous previous studies have demonstrated the suitability of using gene co-expression networks for functional discoveries (Butte and Kohane, 2000; Zhu et al., 2005b). Different approaches to estimating correlation matrix, of testing the significance of these correlations, and of controlling the error rate have been proposed. We emphasize that our goal is to estimate the correlation matrix with reduced variance and improved accuracy.

Towards this goal, the major improvement that we have made is that we provided a full Bayesian treatment that combines correlation estimation and significance testing. For estimation, we improve over existing approaches by providing a regularized full Bayesian estimation. For the hypothesis test, the main improvement over the existing approaches is that we test whether the magnitude correlation is different from a non-zero threshold. This allows for more stringent control of biological significance. For example, in small-sample data the traditional test declares many small but statistically significant correlations to be 
Untransformed Posterior Distribution $(\mathrm{N}=20)$

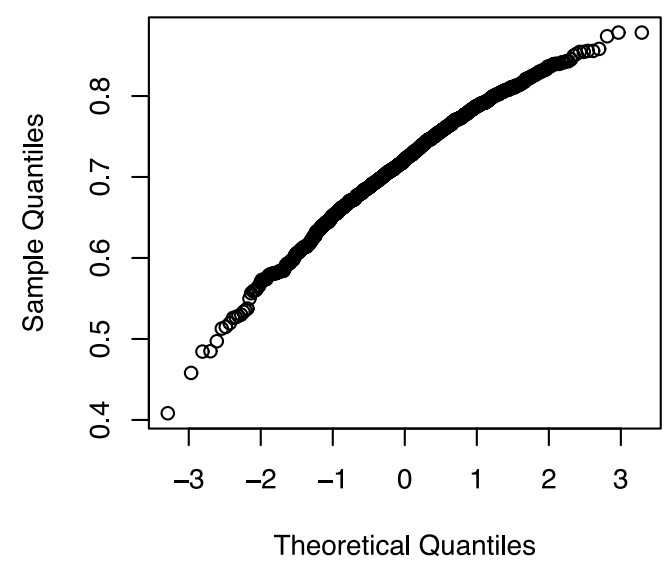

Untransformed Posterior Distribution $(\mathrm{N}=4)$

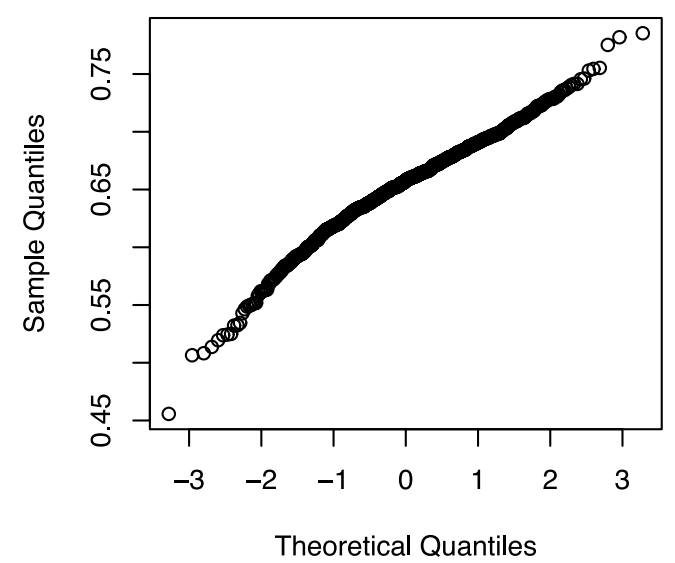

Transformed Posterior Distribution $(\mathrm{N}=20)$

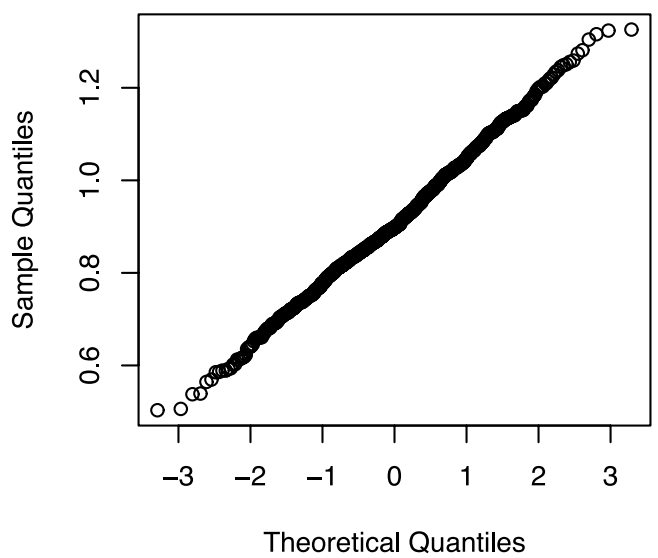

Transformed Posterior Distribution $(\mathrm{N}=4)$

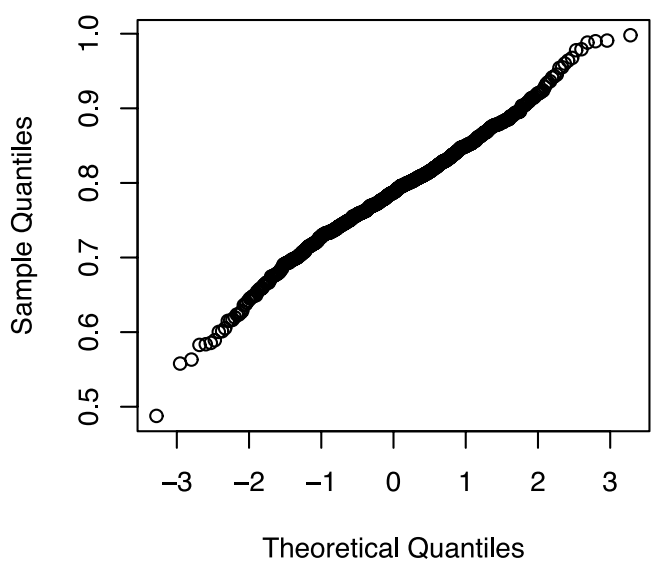

FIG. S-2. Transformed and untransformed posterior distributions at small and large sample size (Example 2).

biologically relevant. However, these may be caused by non-biological effects such as spatial and positional effects of genes along the chromosome (Kluger et al., 2003).

Our framework is sufficiently general to be extended to many different correlation measures, such as full order (Schafer and Strimmer, 2005a) and limited order (Fuente et al., 2004) partial correlation statistics. The rational is that these correlation statistics are asymptotically normal distributed through transformations (Hotelling, 1953). Our approach is also not computationally cumbersome. In deriving the posterior distributions of the correlation "parameters," the conjugate prior and likelihood (i.e., Gaussian parental distribution) were assumed in order to keep the posterior distributions in a closed form. The computational load is thus greatly reduced, making the application to larger networks feasible.

As discussed in Zhu et al. (2005a), one should seek a good combination of level of significance and correlation strength. The Bayesian approach prescribed here imposes a model of the parameters as random variables sampled from a parental population distribution. This model structure allows the regularization of variances by introducing dependency between the parameters. Using simulations, we have shown the superior performance of the Bayesian hierarchical model approach to frequentist estimation approach, in terms of width of the CIs, MSE, and variance, especially for small sample size. The posterior distribution provides a natural way of correlation thresholding that bridges between statistical correlation and biological relevancy. 


\section{APPENDIX}

\section{Selecting prior distribution}

We need to show the joint posterior density $p(\Gamma, \alpha, \beta \mid y)$ is improper if we select the hyperprior distribution $p(\beta) \propto \beta^{-1}$, while $p(\Gamma, \alpha, \beta \mid y)$ is proper if we select the hyperprior distribution $p(\beta) \propto 1$. Interested readers may refer to Exercise 2.8 in Gelman et al. (2004).

\section{Deriving posterior distribution $p(\beta \mid y)$}

Here we present the mathematical details for the posterior distribution $p(\beta \mid y)$ as described in Section 2 . The following is adapted from Chapter V of Gelman et al. (2004).

We factor the marginal posterior density of the hyperparameters as follows:

$$
p(\alpha, \beta \mid y)=p(\alpha \mid \beta, y) p(\beta \mid y)
$$

which is equivalent to:

$$
p(\beta \mid y)=\frac{p(\alpha, \beta \mid y)}{p(\alpha \mid \beta, y)}
$$

We then derive $p(\alpha, \beta \mid y)$ and $p(\alpha \mid \beta, y)$, respectively, as follows. For hierarchical model,

$$
p(\alpha, \beta \mid y) \propto p(\alpha, \beta) p(y \mid \alpha, \beta) .
$$

For many problems, the decomposition in Eq. (17) is of no help, since $p(y \mid \alpha, \beta)$ cannot generally be written in closed form. For the Gaussian distribution, the marginal likelihood has a particularly simple form. The marginal distributions of the sample correlation $\widehat{\Gamma}_{\lambda}$ are independent (but not identically distributed) Gaussian:

$$
p\left(\widehat{\Gamma}_{\lambda} \mid \alpha, \beta\right) \propto N\left(\alpha, \sigma_{\lambda}^{2}+\beta^{2}\right) .
$$

Thus, we can write the marginal posterior density as

$$
p(\alpha, \beta \mid y) \propto p(\alpha, \beta) \prod_{\lambda=1}^{\Lambda} N\left(\widehat{\Gamma}_{\lambda} \mid \alpha, \sigma_{\lambda}^{2}+\beta^{2}\right) .
$$

Assume a uniform conditional prior density $p(\alpha \mid \beta)$, and $p(\alpha \mid \beta, y)$ is Gaussian, i.e.,

$$
p(\alpha \mid \beta, y) \propto N\left(\hat{\alpha}, V_{\alpha}\right),
$$

with

$$
\hat{\alpha}=\frac{\sum_{\lambda=1}^{\Lambda} \frac{1}{\sigma_{\lambda}^{2}+\beta^{2}} \widehat{\Gamma}_{\lambda}}{\sum_{\lambda=1}^{\Lambda} \frac{1}{\sigma_{\lambda}^{2}+\beta^{2}}},
$$

and

$$
V_{\alpha}^{-1}=\sum_{\lambda=1}^{\Lambda} \frac{1}{\sigma_{\lambda}^{2}+\beta^{2}}
$$


Here, $\hat{\alpha}$ is a precision-weighted average of $\Gamma \mathrm{s}$ and $V_{\alpha}$ is the total precision. We define precision as inverse of variance. From Eqs. (16), (19), and (20),

$$
\begin{aligned}
p(\beta \mid y) & =\frac{p(\alpha, \beta \mid y)}{p(\alpha \mid \beta, y)} \\
& \propto \frac{p(\beta) \prod_{\lambda=1}^{\Lambda} N\left(\Gamma_{\lambda} \mid \alpha, \sigma_{\lambda}^{2}+\beta^{2}\right)}{N\left(\alpha \mid \hat{\alpha}, V_{\alpha}\right)}
\end{aligned}
$$

This identity holds for any value of $\alpha$; in particular, it holds if we set $\alpha$ to $\hat{\alpha}$, which makes evaluation of the expression quite simple.

$$
\begin{aligned}
p(\beta \mid y) & \propto \frac{p(\beta) \prod_{\lambda=1}^{\Lambda} N\left(\widehat{\Gamma}_{\lambda} \mid \hat{\alpha}, \sigma_{\lambda}^{2}+\beta^{2}\right)}{N\left(\hat{\alpha} \mid \hat{\alpha}, V_{\alpha}\right)} \\
& \propto p(\beta) V_{\alpha}^{1 / 2} \prod_{\lambda=1}^{\Lambda}\left(\sigma_{\lambda}^{2}+\beta^{2}\right)^{-1 / 2} \exp \left(-\frac{\left(\widehat{\Gamma}_{\lambda}-\widehat{\alpha}\right)^{2}}{2\left(\sigma_{\lambda}^{2}+\beta^{2}\right)}\right),
\end{aligned}
$$

where $\hat{\alpha}$ and $V_{\alpha}$ are defined in Eqs. (21) and (22). Both expressions are functions of $\beta$, which means that $p(\beta \mid y)$ is a complicated function of $\beta$.

\section{On implementations and computational complexities}

Overall, the proposed simulation procedure is computationally inexpensive. The step of drawing $\beta$ from $p(\beta \mid y)$ using the inverse cumulative density function (CDF) method requires iterative computation:

- (Numerically) Scale $p(\beta \mid y)$ (Eq. 3) into a probability distribution, i.e., $P(\beta \mid y)$ so that $\int P(\beta \mid y)=1$.

- (Numerically) Calculate the CDF of the $P(\beta \mid y)$, denoted, $\mathcal{P}(\beta \mid y)$.

- Draw $X$ from $\mathcal{P}(\beta \mid y)$ using inverse CDF method, i.e., $X=\mathcal{P}^{-1}(U) \sim \mathcal{P}$, where $U$ is drawn from $[0,1]$ bounded uniform distribution.

- Re-scale to obtain random draws from $p(\beta \mid y)$.

Each of first three steps require $n$ iterations.

Drawing $\alpha$ from $p(\alpha \mid \beta, y)$ and drawing $\Gamma_{\lambda}$ from $p\left(\Gamma_{\lambda} \mid \alpha, \beta, y\right)$ do not require iteration since they follow normal distributions specified in Eqs. (7) and (8) and can be drawn directly.

\section{ACKNOWLEDGMENTS}

We thank Arcady Mushegian for helpful discussions. We would like to thank anonymous reviewers for their help in improving the quality of this manuscript.

\section{REFERENCES}

Benjamini, Y., and Yekutieli, D. 2005. False discovery rate adjusted multiple confidence intervals for selected parameters. J. Am. Statist. Assoc. 100, 71-80.

Berg, J.M., Tymoczko, J.L., and Stryer, L. 2006. Biochemistry. W.H. Freeman, New York.

Bickel, P.J., and Doksum, K.A. 2000. Mathematical Statistics: Basic Ideas and Selected Topics, 2nd ed. Prentice Hall, Upper Saddle River, NJ.

Breiman, L. 2000. Bagging predictors. Machine Learn. 24, 123-140.

Butte, A., and Kohane, I.S. 2000. Mutual information relevance networks: functional genomic clustering using pairwise entropy measurements. Pacif. Symp. Biocomput. 5, 415-426.

Fisher, R.A. 1923. On the probable error of a coefficient of correlation deduced from a small sample. Metron 1, 1-32.

Fuente, A., Bing, N., Hoeschele, I., et al. 2004. Discovery of meaningful associations in genomic data using partial correlation coefficients. Bioinformatics 20, 3565-3574. 
Gelman, A., Carlin, J.B., Stern, H.S., et al. 2004. Bayesian Data Analysis. Chapmann \& Hall/CRC, Boca Raton, FL. Hastie, T., Tibshirani, R., and Friedman, J. 2001. The Elements of Statistical Learning. Springer, New York.

Hotelling, H. 1953. New light on the correlation coefficient and its transforms. J. R. Statist. Soc. B 15, $193-232$.

Ideker, T., Thorsson, V., Siegel, A.F., et al. 2000. Testing for differentially-expressed genes by maximum-likelihood analysis of microarray data. J. Comput. Biol. 7, 805-817.

Kluger, Y., Yu, H., Qian, J., et al. 2003. Relationship between gene co-expression and probe localization on microarray slides. BMC Genom. 4, 49.

Ledoit, O., and Wolf, M. 2004. A well-conditioned estimator for large dimensional covariance matrices. J. Multiv. Anal. 88, 365-411.

Schafer, J., and Strimmer, K. 2005a. An empirical Bayes approach to inferring large-scale gene association networks. Bioinformatics 21, 754-764.

Schafer, J., and Strimmer, K. 2005b. A shrinkage approach to large-scale covariance matrix estimation and implications for functional genomics. Statist. Appl. Genet. Mol. Biol. 4, 32.

Wieczorke, R., Krampe, S., Weierstall, T., et al. 1999. Concurrent knock-out of at least 20 transporter genes is required to block uptake of hexoses in Saccharomyces cerevisiae. FEBS Lett. 464, 123-128.

Yeung, K.Y., Fraley, C., Murua, A., et al. 2001. Model-based clustering and data transformations for gene expression data. Bioinformatics 10, 977-987.

Zhu, D., Hero, A.O., Qin, Z.S., et al. 2005a. High throughput screening of co-expressed gene pairs with controlled false discovery rate (FDR) and minimum acceptable strength (MAS). J. Comput. Biol. 12, 1029-1045.

Zhu, D., Hero, A.O., Cheng, H., et al. 2005b. Network constrained clustering for gene microarray data. Bioinformatics 21, 4014-4021.

Address reprint requests to:

Dr. Dongxiao Zhu

Stowers Institute for Medical Research 1000 E. 50th St.

Kansas City, MO 64112

E-mail: doz@stowers-institute.org 
This article has been cited by:

1. Q. J. Wang, D. E. Robertson, F. H. S. Chiew. 2009. A Bayesian joint probability modeling approach for seasonal forecasting of streamflows at multiple sites. Water Resources Research 45:5. . [CrossRef] 